\title{
FATORES PREDITIVOS DE COLEDOCOLITÍASE EM DOENTES COM PANCREATITE AGUDA BILIAR
}

\author{
José Gustavo Parreira, Ronaldo Elias Carnut Rego, Tercio De Campos*, \\ Cristina Hachul Moreno, Adhemar Monteiro Pacheco Jr, Samir Rasslan \\ Trabalho realizado no Serviço de Emergência e no Grupo de Vias Biliares e Pâncreas do Departamento \\ de Cirurgia da Faculdade de Ciências Médicas da Santa Casa de São Paulo (FCMSC-SP)
}

RESUMO - OBjetivo. Avaliar o papel da fosfatase alcalina (FA), gama glutamiltransferase $(\gamma \mathrm{GT})$ e ultra-sonografia (US) como fatores preditivos de coledocolitíase em doentes com pancreatite aguda biliar (PAB).

Métodos. Os dados foram coletados prospectivamente durante um período de 31 meses. Quarenta doentes foram incluídos, sendo 30 mulheres, com média etária de $49 \pm 16$ anos. Foram registrados os dados de todos os doentes com pancreatite aguda biliar. Aqueles doentes ictéricos e com a forma grave da doença foram excluídos. As dosagens de FA e GGT, assim como a US, eram realizadas na admissão e 48 horas antes da cirurgia. Todos os pacientes foram submetidos à colangiografia intra-operatória (CIO) ou à colangiografia retrógrada endoscópica (CPRE) préoperatória, que era definida baseada na probabilidade de coledocolitíase. Com 0 intuito de identificar os indicadores de coledocolitíase, as variáveis foram comparadas entre os pacientes com ou sem coledocolitíase. Os testes $t$ de Student, Qui-quadrado e
Fisher foram empregados para a análise estatística, considerando-se $\mathrm{p}<0,05$ como significativo. Os valores preditivos positivo (VPP) e negativo (VPN) foram calculados para cada variável.

ReSULTADOS. Na US realizada à admissão, 15 doentes (37\%) apresentavam dilatação de vias biliares e cinco (12\%), coledocolitíase. Nos exames realizados 48 horas antes da colecistectomia, 34 doentes $(85 \%)$ apresentavam valores elevados de $\gamma \mathrm{GT}$ e $16(40 \%)$ tinham FA acima do normal. Na segunda USG, nove doentes persistiam com dilatação das vias biliares e em três doentes eram vistos cálculos no colédoco. A CPRE pré-operatória foi indicada a 15 doentes (37\%). 0 maior VPP (55\%) foi atribuído à presença de dilatação de vias biliares na US pré-operatória, que também teve o maior VPN (96\%).

ConcLusÃo. A dilatação das vias biliares na US realizada antes da colecistectomia foi o melhor indicador de coledocolitíase.

Unitermos: Pancreatite aguda. Coledocolitíase. Colangiografia.

\section{INTRODUÇÃO}

A pancreatite aguda ocorre em $6 \%$ a $8 \%$ dos portadores de colelitíase sintomática, sendo observada em até $20 \%$ daqueles com microlitíase!. Cerca de $80 \%$ a $85 \%$ das pancreatites são caracterizadas como doença leve, onde a resolução completa é a regra². Destas, um número expressivo desenvolverá novo episódio de pancreatite aguda, sendo que $50 \%$ irão ter novo surto em menos de seis meses $^{3}$. Em conseqüência, preconiza-se a colecistectomia na mesma internação da pancreatite aguda, de preferência por videolaparoscopia 4,5 . Contudo, cerca de $8 \%$ a I $5 \%$ dos portadores de colecistolitíase também apresentam cálculos no colédoco, sendo a freqüência ainda maior logo após o tratamento da pancreatite aguda ${ }^{6-8}$.

*Correspondência:

Rua Dr. Cesário Mota Júnior, 112 Departamento de Cirurgia

CEP: 01221-020 - São Paulo - SP

E-mail: tercio@uol.com.br
Uma opção para o diagnóstico e tratamento dos cálculos remanescentes na via biliar principal, após a inflamação aguda do pâncreas, é a colangiografia retrógrada endoscópica (CPRE) com papilotomia, previamente à colecistectomia videolaparoscópica, não sendo, porém, um procedimento inócuo ${ }^{9}$. Além do que, muitos doentes seriam submetidos à papilotomia desnecessária, caso esta fosse indicada para todos aqueles com pancreatite aguda biliar ${ }^{10}$. Portanto, acredita-se que a CPRE pré-operatória deva ser de indicação seletiva e realizada apenas nos doentes com maior probabilidade de apresentar cálculos no colédoco 8,11,12.

Vários parâmetros já foram pesquisados como fatores preditivos de coledocolitíase, como a dosagem sérica de bilirrubinas, transaminases, amilasemia, assim como a ultra-sonografia abdominal (US) $)^{13,14}$. Especialmente após um episódio de pancreatite aguda, todos estes parâmetros podem estar alterados, mesmo nos doentes anictéricos, dificultando ainda mais a identificação dos candidatos à CPRE pré-operatória ${ }^{10}$.
As enzimas canaliculares por sua vez têm grande sensibilidade porém baixa especificidade $^{8}$. A análise da fosfatase alcalina (FA) e gama glutamiltransferase $(\gamma \in T)$ mostra que elas estão associadas à coledocolitíase em situações eletivas ${ }^{15}$.

O objetivo deste estudo é avaliar os papéis das dosagens séricas de FA e $\gamma \mathrm{GT}$, bem como o da US, realizados na admissão e 48 horas antes da colecistectomia como fatores preditivos de coledocolitíase em doentes com pancreatite aguda biliar.

\section{MÉtodos}

Realizou-se um estudo prospectivo em 3 I meses consecutivos, avaliando os doentes admitidos com pancreatite aguda biliar leve no Serviço de Emergência da Santa Casa de São Paulo. Quarenta doentes foram incluídos no estudo, sendo 30 do sexo feminino. A idade variou de 19 a 77 anos, com média etária de $49 \pm 16$ anos. 0 diagnóstico fez-se através da história clínica, 
Parreira jG et al.

aumento de no mínimo cinco vezes da concentração sérica da amilase e a presença de colecistolitíase na US de abdome. A FA e a $\gamma$ GT também eram dosadas na admissão. 0 quadro de doença leve foi baseado na ausência de complicações locais ou sistêmicas. $O$ tratamento incluiu jejum, analgesia e reposição endovenosa de líquidos e eletrólitos.

Foram incluídos no estudo somente os doentes anictéricos que apresentaram resolução do quadro de pancreatite aguda sem intercorrências, alcançando melhora dos sintomas e normalização da amilasemia. Dois dias antes da data programada para a colecistectomia, realizava-se nova dosagem de FA e $\gamma \mathrm{GT}$, e repetição da US de abdome. A indicação da CPRE pré-operatória baseou-se no aumento da concentração sérica das enzimas canaliculares, bem como na presença de dilatação de vias biliares na US do abdome. Os doentes que se apresentavam com estes dois critérios eram submetidos à CPRE. Os valores considerados normais para $\gamma G T$ foram até $35 \mathrm{U} / \mathrm{dl}$ para doentes do sexo feminino e até $50 \mathrm{U} / \mathrm{dl}$ nos do sexo masculino. A dosagem sérica de FA superior a $250 \mathrm{U} / \mathrm{dl}$ foi considerada elevada. Interpretou-se como dilatadas as vias biliares extra-hepáticas de diâmetro superior a 0,9 cm.

Nos que realizaram CPRE pré-operatória, a papilotomia foi indicada seletivamente naqueles com coledocolitíase ou esvaziamento retardado do contraste da via biliar. Nos demais, durante a colecistectomia, foi realizada a colangiografia. Foram analisados os dados demográficos, freqüência de coledocolitíase e o tratamento. Idade, concentração sérica de FA e yGT à admissão e pré-operatória, bem como alterações ultra-sonográficas à admissão e no pré-operatório, foram pesquisadas como indicadores de coledocolitíase.

Este protocolo já era empregado no tratamento dos doentes com pancreatite aguda biliar, não sendo modificado para a realização deste estudo, e sendo aprovado pelo Comitê de Ética e Pesquisa do Departamento de Cirurgia da Faculdade de Ciências Médicas da Santa Casa de São Paulo.

Empregaram-se os testes $t$ de Student, Qui-quadrado e Fisher para a análise estatística, considerando-se $p<0,05$ como significativo. Para cada variável, calculou-se os valores preditivos positivo e negativo para a detecção de coledocolitíase.

\section{Quadro I - Opções terapêuticas da pancreatite aguda biliar leve com coledocolitíase}

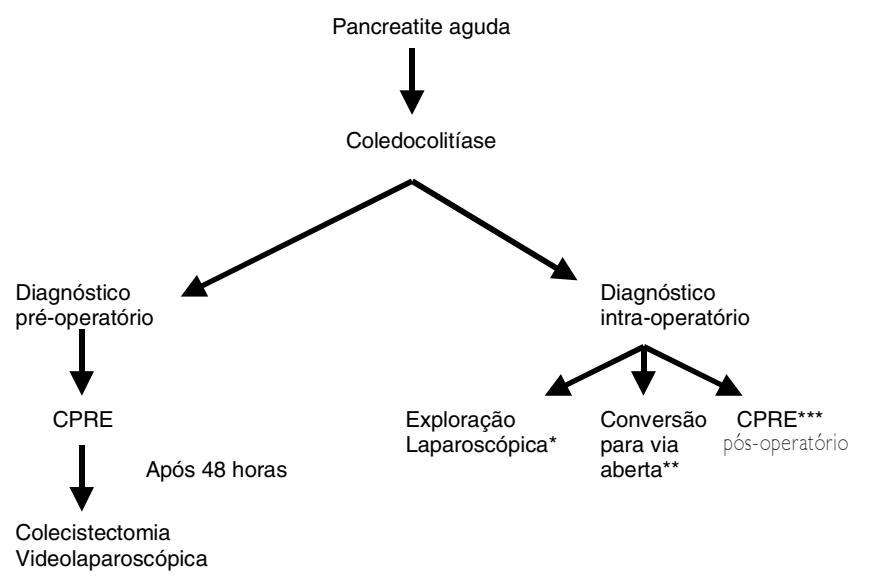

* se tiver condições e recursos

**evitar conversão para exploração das vias biliares em doentes com via biliar fina

****isco de não remover o cálculo

\section{Resultados}

O tempo entre o início dos sintomas e a internação no Serviço de Emergência variou de um a seis dias, em média 2,5 $\pm 1,4$ dia. À admissão, a dosagem sérica de amilase esteve entre 540 e $8108 \mathrm{U} / \mathrm{L}$, em média | 47 | \pm | 522 U/L. No exame ultra-sonográfico do abdome realizado à admissão, 15 doentes (37\%) apresentavam dilatação de vias biliares e cinco (12\%), coledocolitíase. Quatro doentes referiam internação anterior em outra instituição por pancreatite aguda.

Nos exames realizados 48 horas antes da data provável da colecistectomia, a yGT variou de 14 a $615 \mathrm{U} / \mathrm{L}$, com média de $219 \pm 179 \mathrm{U} /$ $\mathrm{dL}$ e a FA esteve entre 83 a $885 \mathrm{U} / \mathrm{dL}$, em média 272 土 $125 \mathrm{U} / \mathrm{dL}$. Trinta e quatro (85\%) apresentavam valores de $\gamma \mathrm{GT}$ e 16 (40\%) de FA acima do normal. Na nova US, nove doentes persistiram com dilatação das vias biliares e em três doentes foram vistos cálculos no colédoco.

A CPRE pré-operatória foi indicada em 15 doentes (37\%) (Figura I). Destes, cinco (33\%) apresentavam cálculos no colédoco, que foram retirados com sucesso em quatro (80\%). Foram realizadas II papilotomias endoscópicas. Dos outros 25 doentes levados à colecistectomia sem CPRE prévia, em apenas um (4\%) foi detectado coledocolitíase pela colangiografia intra-operatória. A colecistectomia por videolaparoscopia foi realizada em 30 doentes e a laparotomia a Kocher nos dez restantes. Nos que iniciaram a operação por via videolaparoscópica, a conversão foi necessária em dois (6\%); um por dificuldade técnica e outro para a coledocotomia e tratamento da coledocolitíase. Houve complicações pós-operatórias em dois doentes, ambos desenvolvendo atelectasia. Não houve óbitos.

A dilatação de vias biliares no exame préoperatório esteve associada significativamente com a presença de coledocolitíase (Tabela I). Microlitíase, dilatação das vias biliares ou mesmo coledocolitíase detectadas na US à admissão não foram fatores relacionados com a presença de coledocolitíase no momento da colecistectomia do mesmo modo que $\mathrm{O}$ aumento da concentração sérica pré-operatória da FA e $\gamma \mathrm{GT}$ (Tabela I). Idade, tempo do início dos sintomas até a colecistectomia e tempo entre internação e colecistectomia não se associaram com a presença de coledocolitíase (Tabela 2).

O maior valor preditivo positivo encontrado foi a dilatação de vias biliares na US pré-operatória, com 55\% (Tabela 3). Isoladamente, a ausência desta variável teve um valor preditivo negativo de $96 \%$. A associação de alterações das enzimas canaliculares e ultrasonográficas não elevou os valores preditivos positivo ou negativo.

\section{Discussão}

A realização da colecistectomia nos doentes com pancreatite aguda biliar é de fundamental 


\begin{tabular}{|c|c|c|c|}
\hline \multicolumn{4}{|c|}{ Tabela I - Indicadores de coledocolitíase } \\
\hline Variável & Presença na amostra & Coledocolitíase & p \\
\hline yGTelevada & $34(85 \%)$ & $4,7 \%$ & 1.000 \\
\hline FAelevada & $16(40 \%)$ & $18,8 \%$ & 0,65 \\
\hline Microlitíase internação & $19(47 \%)$ & $10 \%$ & 0,46 \\
\hline Microlitíase pré-operatória & $13(33 \%)$ & $7 \%$ & 0,35 \\
\hline Dilatação VB internação & $15(37 \%)$ & $26 \%$ & 0,11 \\
\hline Dilatação VB pré-operatória & $9(22 \%)$ & $55 \%$ & $<0,00$ \\
\hline Coledocolitíase US internação & $6(15 \%)$ & $16 \%$ & 0,9 \\
\hline
\end{tabular}

$\gamma \mathrm{GT}$ : Gama glutamiltranspeptidase

FA: Fosfatase alcalina

VB: Vias billares

US: Ultra-som abdominal

\begin{tabular}{|c|c|c|c|}
\hline \multicolumn{4}{|c|}{ Tabela 2 - Comparação entre as médias das variáveis numéricas } \\
\hline Variável & $\begin{array}{c}\text { Coledocolitíase } \\
\text { (6) }\end{array}$ & $\begin{array}{c}\text { Sem coledocolitíase } \\
\text { (34) }\end{array}$ & $P$ \\
\hline $\begin{array}{l}\text { Idade } \\
\text { yGT pré-operatória } \\
\text { FApré-operatória } \\
\text { Tempointernação-cirurgiala } \\
\text { Tempo início-cirurgía }\end{array}$ & $\begin{array}{c}50 \text { anos } \\
347 \pm 216 \\
384 \pm 24 \\
9 \pm 2 \text { dias } \\
11 \pm 1 \text { dias }\end{array}$ & $\begin{array}{c}48 \text { anos } \\
201 \pm 166 \\
252 \pm 38 \\
8 \pm 2 \text { dias } \\
11 \pm 3 \text { dias }\end{array}$ & $\begin{array}{l}0,81 \\
0,06 \\
0,06 \\
0,33 \\
0,73\end{array}$ \\
\hline
\end{tabular}

VGT: Gama glutamiltranspeptidase

FA: Fosfatase alcalina

\begin{tabular}{|c|c|c|}
\hline & & \\
\hline 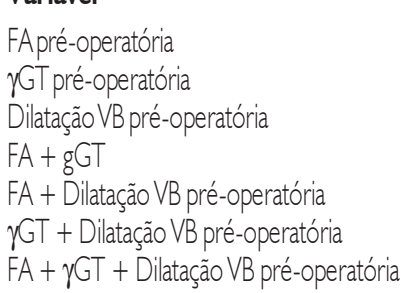 & $\begin{array}{l}20 \\
14 \\
55 \\
20 \\
40 \\
50 \\
40\end{array}$ & $\begin{array}{l}88 \\
83 \\
96 \\
83 \\
100 \\
100 \\
100\end{array}$ \\
\hline
\end{tabular}

VPP: Valor preditivo positivo; VPN: Valor preditivo negativo; FA: Fosfatase alcalina alterada;

VGT: Gama glutamiltransferase alterada; VB: Vias billares; Pré-op.: pré-colecistctomia.

importância no intuito de prevenir novos surtos, devendo ser realizada de preferência na mesma internação $0^{4}$. A videolaparoscopia é a via de eleição para a colecistectomia, sendo que nesta casuística não houve complicações relacionadas ao procedimento, e com uma necessidade de conversão para laparotomia em apenas $6 \%$ dos doentes.

No entanto, há controvérsia quanto à melhor forma de tratamento da coledocolitíase na era da cirurgia minimamente invasiva. As opções em questão incluem a CPRE com papilotomia previamente à colecistectomia, podendo ainda ser realizada no pósoperatório ${ }^{16-18}$; a coledocotomia com retirada dos cálculos por videolaparoscopia 19-22, ou mesmo a conversão da operação para laparotomia e coledocotomia por via aberta ${ }^{23}$.

A resolução da coledocolitíase pela via laparoscópica representa aumento do tempo operatório da mesma forma que a resolução pela via aberta, porém, com necessidade de equipamentos especializados ${ }^{24}$. A coledocotomia aumenta também a morbidade, pela necessidade de drenagem da via biliar com o dreno de Kehrr ${ }^{24,25}$.

Já a CPRE pré-operatória permitiria 0 tratamento da coledocolitíase sem estes problemas. Contudo, sua indicação em todos os doentes levaria a uma freqüência muito alta de exames desnecessários, com custo e morbidade dispensáveis ${ }^{11,12,26}$. Podemos ter complicações em até $10 \%$ dos casos, sendo as principais o agravamento ou recidiva da pancreatite aguda, o sangramento, a perfuração duodenal e a colangite. A mortalidade pode chegar de $0,37 \%$ a $\mid \%{ }^{16,27}$. Sugiyama et al., em 2003, constataram um aumento da morbidade em doentes submetidos à CPRE e dilatação da papila com balão. Este aumento ocorreu principalmente nos doentes jovens, com história de pancreatite, com via biliar fina e quando a canulação se mostrava difícil'.

Dos nossos 15 doentes que fizeram CPRE no pré-operatório, cinco tinham cálculos e I| foram submetidos à papilotomia, sendo que seis doentes tiveram uma papilotomia desnecessária. Não tivemos complicações relacionadas à CPRE.

Por outro lado, Neoptolemos et al., em 1988, relataram benefício da realização da CPRE nas primeiras 72 horas de internação em doentes com pancreatite aguda de origem biliarr $^{28}$. Admite-se, porém, que a indicação da CPRE na pancreatite aguda seja bem estabelecida nos doentes com elevação das bilirrubinas ou naqueles com colangite associada à pancreatite 4 .

Deve-se ainda considerar o fato de que muitos hospitais não dispõem de CPRE. Seu custo, quando analisado, atinge valores altos: uma CPRE diagnóstica custa em média U\$940, subindo para $\cup \$ 1060$ quando utilizada para o tratamento da coledocolitíase ${ }^{29}$.

Na reunião da International Association of Pancreatology para elaboração de um guia para - tratamento da pancreatite aguda, realizada em Heidelberg em 2002, não ficou definida nenhuma recomendação para o uso sistemático da CPRE na pancreatite aguda, devido ao fato de que dois estudos prospectivos randomizados, um inglês e outro de Hong Kong, mostraram benefícios da CPRE, enquanto que outro alemão concluiu o contrário ${ }^{4}$.

Com nossos critérios, obtivemos apenas $33 \%$ de coledocolitíase dentre os doentes que foram submetidos à CPRE. Procuram-se, então, critérios para a indicação seletiva da CPRE pré-operatória.

Vários critérios já foram pesquisados na tentativa de identificar os doentes com maior chance de coledocolitíase, pois desta forma a CPRE teria resultados mais efetivos nestes casos. A presença de icterícia persistente é um forte indicador de coledocolitíase, e, por este motivo, os doentes ictéricos foram excluídos do estudo. 
PARREIRA JG et AL.

Considerando-se doentes com litíase biliar em geral, a dosagem de FA e $\gamma \mathrm{GT}$ tem sido útil na identificação daqueles com maior probabilidade de coledocolitíase ${ }^{8}$. Neste estudo, notamos que as enzimas canaliculares apresentaram-se elevadas na maioria dos doentes após o episódio de pancreatite aguda. Desta forma, não foram critérios efetivos na seleção dos candidatos para CPRE. O melhor indicador de coledocolitíase obtido nesta amostra foi a dilatação das vias biliares ao exame ultra-sonográfico realizado 48 horas antes da colecistectomia. Este achado esteve associado à coledocolitíase em 55\% dos casos. É importante ressaltar que a dilatação das vias biliares ou mesmo a presença de coledocolitíase na ultra-sonografia abdominal realizada na admissão apresentaram associação com coledocolitíase no momento da colecistectomia em não mais que $26 \%$ dos doentes.

Outro ponto a ser discutido é o melhor momento para a operação. Liu et al., em 1997. relataram que se a colecistectomia for realizada logo na admissão a necessidade de exploração das vias biliares por coledocolitíase chega a $70 \%$, contra I $5 \%$ a $20 \%$ após o quinto dia do surto 30 .

Desta forma, acreditamos que a CPRE deva ser de indicação seletiva e reservada apenas para os doentes com maior probabilidade de apresentar cálculos no colédoco.

Existem algumas formas de tratamento da pancreatite aguda biliar leve (Quadro I): quando se tem o diagnóstico de coledocolitíase pré-operatório, pode-se fazer a CPRE com papilotomia para retirada deste cálculo e, após 48 horas, programa-se a colecistectomia videolaparoscópica, caso o doente não tenha apresentado nenhuma intercorrência relacionada à CPRE. Quando o diagnóstico de coledocolitíase é feito durante a colecistectomia, três condutas são possíveis: exploração por videolaparoscopia das vias biliares, caso haja equipamento disponível; retirada do cálculo no pós-operatório, especialmente naqueles cálculos menores que $1 \mathrm{~cm}$ e desde que se tenha um endoscopista experiente disponível; ou a conversão para a cirurgia aberta com exploração das vias biliares.

No entanto, a conversão deve ser evitada quando o colédoco for fino, pelo risco decorrente da sua exploração, em especial a estenose cicatricial. Por outro lado, quando se opta pela extração do cálculo por CPRE no pós-operatório, existe o risco de insucesso do procedimento e, com isso, a necessidade de nova intervenção.

\section{Conclusão}

A análise deste trabalho mostra que a dilatação das vias biliares à ultra-sonografia abdominal realizada 48 horas antes da data prevista para colecistectomia foi o melhor indicador de coledocolitíase.

\section{Conflito de interesse: não há.}

\section{SUMMARY}

\section{Predictors of CHOledocholithiasis IN PATIENTS SUSTAINING ACUTE BILIARY PANCREATITIS}

BACKGROUND. To assess the role of alkaline phosphatase (AP), gamil-glutamyltransferase (gGT) and abdominal ultrasound (US) as predictors of choledocholithiasis in patients sustaining acute biliary pancreatitis.

METHODS. Data was prospectively collected during a period of 31 months. Forty patients were included, 30 were female and the meanage was $49 \pm$ 16. All patients sustaining acute biliary pancreatitis were enrolled. Patients with clinical jaundice and severe pancreatitis were excluded. Serum content of AP and gGT as wellas US were assessed at admission and 48 hours before cholecistectomy. All patients underwent intraoperative cholangiography (IOC) or preoperative endoscopic retrograde cholangiography (ERCP), which was indicated based on the odds of choledocholithiasis. In order to identify the predictors of choledocholithiasis, variables were compared between patients sustaining or not such alteration in cholangiography. Student t, Fisher and chi square tests were used for statistical analysis, considering $p<0.05$ as significant. Positive (PPV) and negative predictorvalues (NPV) were calculated for each variable.

RESULTS. Uponadmission, 15(37\%) patients sustained biliary tract dilatation and 5 (12\%) choledocholithiasis at the US. Forty eight hours before the operation, 34 (85\%) patients had altered levels of gGT and 16 (40\%) of AP. Preoperative US showed biliary tract dilatation in nine patients and choledocholithiasis in three. ERCP was performed in 15(37\%) cases. Higher PPV (55\%) was attributed to pre-operative US, which had also a NPV of $96 \%$.
CONCLUSION. The best predictor of choledocholithiasis in patients sustaining mild acute pancreatitis was the biliary tract dilatation in pre-operative US. [Rev Assoc Med Bras 2004; 50(4): 39|-5]

KEY WORDS: Acute pancreatitis. Choledocholithiasis. Cholangiography.

\section{ReferênCIAS}

I. Neoptolemos JP. Endoscopicsphincterotomy in acute gallstone pancreatitis. Br J Surg 1993; 80:547-9

2. Werner J, Hartwig W, Uhl W, Muller C, Buchler MW. Useful markers for predicting severity and monitoring progression os acute pancreatitis. Pancreatology 2003; 3: I I 5-27.

3. Pellegrini CA. Surgery for gallstone pancreatitis. Am J Surg 1993; 165:5 I 5-8.

4. Uhl W, Warshaw A, Imrie C, Bassi C, McKay C), Lankisch PG, et al. IAP guideliness for the surgical management of acute pancreatitis. Pancreatology 2002; 2:565-73.

5. McCullough LK, Sutherl FR, Preshaw R, Kim S. Gallstone pancreatitis: does discharge and readmission for cholecystectomy affect outcome? HPB 2003; 5:96-9.

6. Basso N, Pizzuto G, Surgo D, Materia A, Silecchia G, Fantini A, et al. Laparoscopic cholecystectomy and intraoperative endoscopic sphincterotomy in the treatment of cholecysto-choledocholithiasis. Gastrointest Endosc 1999; 50:532-5.

7. Menezes N, Marson LP, Debeaux AC, Muir $I M$, Auld CD. Prospective analysis of a scoring system to predict choledocholithiasis. Br J Surg 2000; 87: | | 176-81.

8. De Campos T. Análise de fatores clínicos, bioquímicos e ultra-sonográficos preditivos de coledocolitíase em doentes portadores de litíase vesicular avaliados por colangiografia [dissertação]. São Paulo: Faculdade de Ciências Médicas, Santa Casa de São Paulo; 2002

9. Sugiyama M, Izumisato Y, Abe N, Masaki T, Mori T, Atomi Y. Predictive factors for acute pancreatitis and hyperamylasemia after endoscopic papillary balloon dilation. Gastrointest Endosc 2003; 57:531-5.

10. Silva RA, Ueda RYY, Rego REC, Pacheco Jr AM, Fava J, Rasslan S. Tratamento cirúrgico postergado da pancreatite aguda biliar. Rev Col Bras Cir 2000; 27: I67-72.

11. Cotton PB, Baillie J, Pappas TN, Meyers WS Laparoscopic cholecystectomy and the biliary endoscopist. GastrointestEndosc 1991; 37:94-6.

12. Clair DG, Carr-Locke DL, Becker JM, Brooks DC. Routine cholangiography is not warranted during laparoscopic cholecystectomy. Arch Surg 1993; 128:55।-5.

13. Abboud PC, Malet PF, Berlin JA, Staroscik R, Cabana MD, Clarke JR, et al. Predictors of common bile duct stones prior to cholecystectomy: a meta-analysis. Gastrointest Endosc 1996; 44:450-7. 
14. Liu TH, Consorti ET, Kawashima A, Ernst RD, Black CT, Greger PH, et al. The efficacy of magnetic resonance cholangiography for the evaluation of patients with suspected choledocholithiasis before laparoscopic cholecystectomy. Am J Surg 1999; 178:480-4.

15. Wang CH, MO LR, Lin RC, Kuo JY, Chang KK. Rapid diagnosis of choledocholithiasis using biochemical tests in patients undergoing laparoscopic cholecystectomy. Hepatogastroenterology 200 I; 48:6 I8-2I.

16. Rosenthal RJ, Rossi RL, Martin, RF. Options and strategies for the management of choledocholithiasis. World J Surg 1998; 22: I I25-32.

17. Hawasli A, Lloyd L, Cacucci B. Management of choledocholithiasis in the era of laparoscopic surgery. Am Surg 2000; 66:425-31.

18. Costi R, lusco DR, Sarli L. Comment on a balanced approach to choledocholithiasis. Surg Endosc 2002; 16:724-5.

19. Suc B, Escat J, Cherqui D, Fourtanier G, Hay J, Fingerhut $A$, et al. Surgery vs endoscopy as primary treatment in symptomatic patients with suspected common bile duct stones. Arch Surg 1998; 133:702-8.

20. Lauter DM, Froines EJ. Laparoscopic common duct exploration in the management of choledocholithiasis. Am J Surg 2000; 179:372-4.
21. Memon MA, Hassaballa H, Memon MI. Laparoscopic common bile duct exploration: the past, the present and the future. Am J Surg 2000; 179:309-15

22. Isla A, Griniatsos J, Rodway A. Single-stage definitive laparoscopic management in mild acute biliary pancreatitis. J Laparoendosc Adv Surg Tech A 2003; 13:77-8|.

23. Moreaux J. Traditional surgical management of common bile duct stones: a prospective study during a 20-year experience. Am J Surg 1995; 169:220-6.

24. Onken JE, Brazer SR, Eisen GM, Williams DM, Bouras EP, Delong ER, et al. Predicting the presence of choledocholithiasis in patients with symptomatic cholelithiasis. Am J Gastroenterol 1996; 91:762-7.

25. Tham TCK, Collins JSA, Watson RGP, Ellis PK, Mcllrath EM. Diagnosis of common bile duct stones by intravenous cholangiography: prediction by ultrasound and liver function tests compared with endoscopic retrograde cholangiography. Gastrointest Endosc 1996; 44: I58-63.

26. Aubertin MD, Prat F, Fritsch J, Choury AD, Etienne JP. Diagnostic ERCP in the era of laparoscopic surgery. Hepatogastroenterology 1995; 42:607-II.

27. Franceschi D, Brandt C, Margolin D, Szopa B,
Ponsky J, Priebe $\mathrm{P}$, et al. The management of common bile duct stones in patients undergoing laparoscopic cholecystectomy. Am Surg 1993; 59:525-32.

28. Neoptolemos JP, Carr-Locke DL, London NJ, Bailey IA, James D, Fossard DP. Controlled trial of urgent endoscopic retrograde cholangiopancreatography and endoscopic sphincterotomy versus conservative treatment for acute pancreatitis due to gallstones. Lancet 1988; 2:979-83.

29. Scheiman JM, Carlos RC, Barnett JL, Elta GH, Nostrant TT, Chey WD, etal. Can endoscopic ultrasound or magnetic resonance cholangiopancreatography replace ERCP in patients with suspected biliary disease? A prospective trial and cost analysis. Am J Gastroenterol 200 I; 96:2900-4

30. Liu CL, Lo CM, Fan ST. Acute biliary pancreatitis: diagnosis and management. World J Surg 1997; 21 : 1 49-54.

Artigo recebido: 10/10/03 Aceito para publicação: 13/04/04 